\title{
Pasteurella multocida meningitis with disseminated intravascular coagulation and multi-organ failure in an adult with no bite history
}

\author{
Edna Juarez MD, Avinash Adiga MD
}

\begin{abstract}
A 70-year-old woman with an extensive past medical history presented to the emergency center with two weeks of generalized weakness, fatigue, resting shortness of breath, and abdominal pain. The patient was bradycardic and hypotensive and was admitted to the intensive care unit. Within a few hours of admission, she developed worsening lethargy and respiratory acidosis and required intubation and vasopressor support. Laboratory results were remarkable for azotemia, anemia, and thrombocytopenia. Magnetic resonance imaging of the brain was unremarkable; an EEG reported mildly generalized slowing and no epileptiform waves. Cerebrospinal fluid culture was positive for Pasteurella multocida. The patient later developed acute hepatic injury, acute kidney injury, and an elevated D-Dimer with a decreased fibrinogen consistent with disseminated intravascular coagulation. After twelve days her family decided for comfort care only; the patient was extubated and died.
\end{abstract}

Keywords: meningitis, Pasteurella multocida, multi-organ failure, disseminated intravascular coagulation

\section{INTRODUCTION}

Gram negative bacteria are an infrequent cause of community-acquired meningitis, accounting for only $3.6 \%$ of the cases; this usually occurs in older patients with chronic debilitating conditions. Pasteurella multocida is one of the most infrequent causes of Gram negative meningitis even in this population group. According to a recent case series report, as of 2015, only 48 cases of Pasteurella multocida associated meningitis have been reported in the literature in both healthy and immunocompromised adults; $26 \%$ of these cases had had recent neurosurgery.

Corresponding author: Edna Juarez

Contact Information: edna.juarez@ttuhsc.edu

DOI: $10.12746 /$ swrccc.v5i19.387
CASE

A 70-year-old woman presented to the emergency center with generalized weakness, fatigue, and shortness of breath at rest for two weeks. She had a past history of transfusion resistant anemia secondary to myelodysplastic syndrome diagnosed six years prior to admission with a bone marrow biopsy. At the time of presentation, the patient had mild chronic anemia and thrombocytopenia treated with iron replacement. She also had congestive heart failure, coronary artery disease status post triple coronary bypass three years prior to admission, hypothyroidism, and diabetes mellitus type 2. The patient had no recent direct exposure to dogs, cats, or poultry. She was bradycardic (heart rate 56 beats per minute) and hypotensive (blood pressure $86 / 36 \mathrm{mmHg}$ ) and was admitted to the intensive care unit. Examination was remarkable for decreased breath sounds bilaterally and crackles in the lung bases; no fever was noted. A few hours 
after admission, she developed worsening lethargy and respiratory acidosis requiring intubation, ventilator support, and vasopressor medications.

Laboratory tests revealed hemoglobin $9.9 \mathrm{gm} / \mathrm{dL}$, hematocrit $30.6 \%$, leucocyte count $5.15 \mathrm{k} / \mu \mathrm{L}$, platelet count $64 \mathrm{k} / \mu \mathrm{L}$, sodium $137 \mathrm{mmol} / \mathrm{L}$, potassium $5.7 \mathrm{mmol} / \mathrm{L}$, BUN $60 \mathrm{mg} / \mathrm{dL}$, creatinine $1.7 \mathrm{mg} / \mathrm{dL}$, total bilirubin $1.1 \mathrm{mg} / \mathrm{dL}$, aspartate transaminase $22 \mathrm{IU} / \mathrm{L}$, alanine transaminase $9 \mathrm{IU} / \mathrm{L}$, alkaline phosphatase $132 \mathrm{IU} / \mathrm{L}, \mathrm{TSH} 5.37 \mu \mathrm{IU} / \mathrm{ml}$, and cortisol $11.5 \mathrm{mcg} / \mathrm{dL}$. Computed tomography scans and magnetic resonance imaging of the brain were unremarkable. An EEG showed mild generalized slowing and no epileptiform waves. After 48 hours the patient's blood pressure stabilized, she was weaned off vasopressors, her mental status slightly improved, and she was extubated. However, following extubation, the patient remained confused. Cerebrospinal fluid studies reported protein $122 \mathrm{mg} / \mathrm{dL}$, glucose $46 \mathrm{mg} / \mathrm{dL}$, $42 \mathrm{WBC} / \mathrm{mm}^{3}$, and a culture positive for Pasteurella multocida. The next day her liver enzymes increased (aspartate transaminase $2945 \mathrm{IU} / \mathrm{L}$, alanine transaminase $1346 \mathrm{IU} / \mathrm{L})$, her BUN increased to $58 \mathrm{mg} / \mathrm{dL}$, and her creatinine increased to $1.8 \mathrm{mg} / \mathrm{dL}$. The D-Dimer was $5883 \mathrm{ng} / \mathrm{ml}$ and fibrinogen level was $171 \mathrm{mg} / \mathrm{dl}$, consistent with disseminated intravascular coagulation (DIC). Despite treatment with antibiotics (IV ceftriaxone) and supportive therapy, the patient's condition deteriorated, and her family decided to withdraw care on day 12.

\section{Discussion}

According to Druan et al, Gram negative bacteria are an infrequent cause of community-acquired meningitis and account for only $3.6 \%$ of the cases in the United States. ${ }^{1}$ These infections usually occur in older patients with chronic debilitating diseases. Pasteurella multocida is one of the least common etiologic causes in any population group and is mainly reported in neonates. Pasteurella multocida is a Gram negative, non-motile, non-spore-forming coccobacillus with bipolar staining features. The bacteria typically appear as single bacilli on Gram stain; however, pairs and short chains can also be seen. ${ }^{2}$ These bacteria often exist as commensals in the upper respiratory tracts of many livestock, poultry, and domestic pets, especially cats and dogs. Infection in humans is often associated with an animal bite, scratch, or lick, but infection without epidemiologic evidence of animal contact may occur. ${ }^{3}$ Wound, deep tissue, and more disseminated infections, including endocarditis or meningitis, the latter mimicking Haemophilus influenzae or Neisseria meningitides infections, can develop. ${ }^{4}$ Occasionally infections at the extremes of life has been associated with bacteremia with sepsis, septic shock, and disseminated intravascular coagulation. A recent case series reported that only 48 cases of Pasteurella multocida associated meningitis have been reported in literature in both healthy and immunocompromised adults as of $2015 ; 26 \%$ of these cases had had recent neurosurgery. ${ }^{5}$

The American Pet Products Association estimates that 180 million dogs and cats live in the United States and that cats currently outnumber dogs by 12 million. Animal bites account for $1 \%(300,000)$ of annual emergency department visits and create health care expenditures of approximately $\$ 30$ million per year. Ten percent of animal bites require medical attention; $1-2 \%$ eventually require hospitalization. Ten to twenty human deaths per year occur following an animal bite. Infectious complications develop in $15-20 \%$ of dog-related bites and more than $50 \%$ of cat-related bites. Following a bite, a rapidly progressive cellulitis may develop; deeper structures, including tendons, joints, and bones, can become infected, especially in cat-related injuries. Dissemination can occur. ${ }^{6}$ A survey of literature over the past 30 years suggests that 20 to 30 human deaths due to Pasteurellosis occur annually worldwide, but this rate appears to be rising and nearly all deaths are related to animal bites. Wound infections with Pasteurella multocida tend to be highly aggressive. Skin or soft tissue inflammation, erythema, local lymphadenopathy, fever, pain and swelling develop within the first 24 hours; the overall mortality rate is from 25 to $30 \%$ with neurological complications found in 17 to $29 \%$ of cases. $^{7}$

In 2015 Giordanno published a retrospective study involving 44 cases of Pasteurella multocida infection and observed that infections without a bite 
history seemed to occur in patients with serious comorbidities who often have immunosuppression. This group of patients was more frequently hospitalized, more often required ICU-based care, had longer lengths of stay, and had higher mortality rates than patients with bite-associated infections. The mortality rate for patients with Pasteurella multocida in the absence of an animal bite was 4 of 19 patients $(21 \%)$, and all of these patients appeared to have an underlying disease that could lead an immunocompromised state. $^{8}$ Pasteurella multocida infections are treated primarily with penicillin, ampicillin, and cephalosporins. Although the frequency of resistance to $\beta$-lactams remains low, $\beta$-lactamase-producing strains have been described. ROB-1 is the most frequent enzyme conferring $\beta$-lactam resistance; TEM-1 has been reported from a human isolate in France. More recently, BlaP1 (Pse-1/CARB-2), conferring resistance to ampicillin and carbenicillin, has been described in a Pasteurella multocida strain of avian origin in Taiwan. ${ }^{9}$

Cases like the one we present indicate that a high index of suspicion for Gram negative pathogens, even in the absence of risk factors such animal contact, especially in older and/or debilitated patients, and the empiric use of broad spectrum beta lactam antibiotics while awaiting culture results can potentially decrease mortality, morbidity and hospital length of stay in patients with possible meningitis. Appropriate pet vaccination and universal hygiene measures can also potentially prevent this condition.

Article citation: Juarez E, Avinash Adiga A. Pasteurella multocida meningitis with disseminated intravascular coagulation and multi-organ failure in an adult with no bite history. The Southwest Respiratory and Critical Care Chronicles 2017; 5(19): 22-24

From: Department of Internal Medicine at Texas Tech University Health Sciences Center in Lubbock, TX

Submitted: 3/8/2017

Accepted: 4/14/2017

Reviewer: Edward Pesanti MD

Conflicts of interest: none

\section{REFERENCES}

1. Duran ML, Calderwood SB, Weber DJ, Miller SI, Southwick FS et al. Acute bacterial meningitis in adults. A review of 493 episodes. N Engl J Med 1993 Jan 7; 328(1):21-8.

2. Kuhnert P, Christensen H. Pasteurellaceae: Biology, Genomics and molecular aspects. Cais Academ Press. 2008.

3. Ryan KJ, Ray CG (Editors) 2004. Sherris Med Biol 4th Ed. Mc Graw Hill. Columbus, OH.

4. Christidou A, Maraki S, Gitti Z, Magurean-Zervaki K, Tselentis Y. Human Infections due to Pasteurella multocida in a tertiary care hospital. A 12 year review. Proceeding of the $16^{\text {th }}$ European congress of clinical microbiology and infectious disease. 2006.

5. Bardou M, Honorat E, Dobourg G, Couderc C, Fournier PE, et all. Meningitis caused by Pasteurella multocida in a dog owner, without a dog bite: clonal lineage identification by MALDI-TOF mass spectrometry. BMC Res Notes 2015 Dec $28 ; 8: 826$.

6. Weber DJ, Wolfson JS, Swartz MN, Hooper DC. Pasteurella multocida infections. Report of 34 cases and review of literature. Medicine (Baltimore) 1984 May; 63(3):133-54.

7. Wilson BA, Ho M. Pasteurella multocida: from zoonosis to cellular microbiology. Clin Microbiol Rev 2013 Jul; 26(3):631-55.

8. Giordanno A, Dincman T, Clyburn BE, Steed LL, Rockey DC. Clinical features and outcomes of Pasteurella multocida infection. Medicine (Baltimore) 2015 Sep; 94(36): e1285.

9. San Millan A, Escudero JA, Gutierrez B, Hidalgo L, Garcia N, Llagostera M, Dominguez L, Gonzalez-Zorn B. Multiresistance in Pasteurella multocida is mediated by coexistence of small plasmids. Antimicrob Agents Chemother 2009 Aug; 53(8):3399-404. 